\title{
The sign of the reaction volume for the water/silica reaction
}

\author{
G. Schell, T. Fett
}


IAM Institute for Applied Materials

\section{Impressum}

Karlsruher Institut für Technologie (KIT) www.kit.edu

\section{(c) (1) (2)}

This document is licensed under the Creative Commons Attribution - Share Alike 4.0 International License (CC BY-SA 4.0): https://creativecommons.org/licenses/by-sa/4.0/deed.en

2019

ISSN: 2194-1629 


\begin{abstract}
In the present report, we deal with the question whether the equilibrium of the silica/water reaction shifts under tensile stresses to an increased or reduced hydroxyl concentration or equivalently, which sign the reaction volume shows. From our analysis on the basis of the Le Chatelier-Braun Theorem, it can be concluded that the equilibrium constant of the watersilica reaction is enhanced due to tensile stresses and that the reaction volume change is positive. In additon it will be shown that the apparently found enlarged water solubility under compressive stresses by Nogami and Tomozawa [2] is an artifact of the stress-dependent diffusivity.
\end{abstract}




\section{Contents}

1 Water reaction with silica 1

2 Conclusion from the Theorem of Le Chatelier/Braun 2

3 Negative reaction volume from literature 5

4 Summary 7

$\begin{array}{ll}\text { References } & 8\end{array}$ 


\section{Water reaction with silica}

According to Doremus [1], water penetrates silica glass as molecular water. At temperatures, $T<450^{\circ} \mathrm{C}$, the equilibrium constant, $k$, of the silica/water reaction

$$
\equiv \mathrm{Si}-\mathrm{O}-\mathrm{Si} \equiv+\mathrm{H}_{2} \mathrm{O} \leftrightarrow \equiv \mathrm{SiOH}+\mathrm{HOSi} \equiv
$$

is given by for temperatures $<450^{\circ} \mathrm{C}$ by the following equation [2]:

$$
k_{1}=\frac{S}{C}
$$

where $S$ is the hydroxyl group concentration, [ $\mathrm{SiOH}]$, and $C$ is the concentration of molecular water, $\left[\mathrm{H}_{2} \mathrm{O}\right]$. The reason for the apparent unimolecular reverse reaction of eq.(1) is that the two hydroxyl groups that form during the forward reaction are not independent [2]; they are closely associated with one another and can react easily to form water during the reverse reaction. Above $450^{\circ} \mathrm{C}$, it holds

$$
k_{2}=\frac{S^{2}}{C}
$$

In this case, the hydroxyl groups can move away from one another; they become independent, and the reverse reaction behaves as a normal bimolecular reaction [1,2]. Although water molecules can move freely within silica glass by molecular diffusion [1], the hydroxyl groups, $S$, are immobile [1] at low temperatures. The amount of water tied up in $\mathrm{SiOH}$ is $S / 2$. As defined by Doremus [1], the total water at the surface, $C_{w}$, is given by the sum of the molecular water and the water tied up in $\mathrm{SiOH}$ :

$$
C_{w}=C+\frac{1}{2} S=C\left(1+\frac{1}{2} k\right)
$$

The classic work on the effect of pressure on the equilibrium constant of a chemical reaction was done by Le Chatelier [3]. From his work, it is well known that chemical reactions that exhibit a change in volume will be sensitive to the ambient pressure of the reaction. Changing the pressure changes the equilibrium constant of the reaction and hence the ratio of the concentration of reaction products to reactants.

Using the enhanced value of the equilibrium constant and the following thermodynamic equation [4]:

$$
\frac{\partial \ln k}{\partial p}=-\frac{\Delta \bar{V}}{R T}
$$

It is possible to obtain an estimate of the effective stress at the crack tip during the fracture experiment. In the above equation, $p$ is the pressure applied to the glass at the crack tip, $\Delta \bar{V}$ is the reaction volume, the change in the volume of the glass during the 
chemical reaction, i.e., the volume of the products minus the volume of the reactants during the reaction, $R$ is the universal gas constant and $T$ is the temperature in $K$. The reaction volume is normally assumed to be a constant, but clearly can be pressure dependent, see reference [4] for a discussion of this point.

Equation (5) can also be expressed in terms of the crack tip stresses:

$$
\frac{\partial \ln k}{\partial \sigma_{h}}=\frac{\Delta \bar{V}}{R T}
$$

where $\sigma_{h}$ is the hydrostatic stress at the crack tip.

In applying eq. (5) to mechanical problems, it must be noted that the signing in mechanics differs from that in chemistry. In chemistry, pressures are regarded as positive variables, but are counted as negative stresses in continuum mechanics. This makes it necessary to replace the pressure $p$ in eq. (5) by the hydrostatic stress term $-\sigma_{h}$, defined as the average of the three normal stress components of any stress tensor.

Then the equivalent representation of eq. (6) is given by

$$
k=k_{0} \exp \left[\frac{\sigma_{h} \Delta \bar{V}}{R T}\right]
$$

In literature there exist contrary assumptions and conclusions, in which direction the equilibrium of the reaction eq.(1) must shift, when tensile stresses are applied or equivalently, whether the reaction volume would be positive or negative. Whereas Nogami and Tomozawa [5] conclude a negative reaction volume, Agarwal et al. [6] come to the opposite result, namely $\Delta \bar{V}>0$. The latter result can also be concluded from the positive volume in the derivation of $v-K$-curves on the basis of the reaction rate theory (for an overview see e.g. [7]). In the context with the conclusion in [5] it should be noted, that the observed effect is a consequence of the stress-dependent diffusivity as was outlined in [8]. In section 3, we will address this point. In a newer paper [9], water solubility was discussed again as an increase with applied compressive stress, i.e. $\Delta \bar{V}<0$. In Section 2, we will validate the occurrence of positive reaction volumes, by making use of the universal Theorem by Le Chatelier [3] and Braun [10].

\section{Conclusion from the Theorem of Le Chatelier and Braun}

This finding is rather trivial. When water reacts with silica to hydroxyl $S=[\mathrm{SiOH}]$, a volume expansion occurs as was early reported by Shelby [11], Shackleford [12] and Brückner $[13,14]$. From their measurements a volume swelling strain of the hydroxyl mass concentration $S[15]$ can be derived:

$$
\varepsilon_{v, s w}=\frac{18}{17}(1+\chi) \frac{S}{2}=\kappa \times S
$$


with the proportionality factor

$$
\kappa=0.97[0.92,1.02]
$$

$[16,17,15]$. In (9) the numbers in brackets represent the $95 \%$ confidence interval.

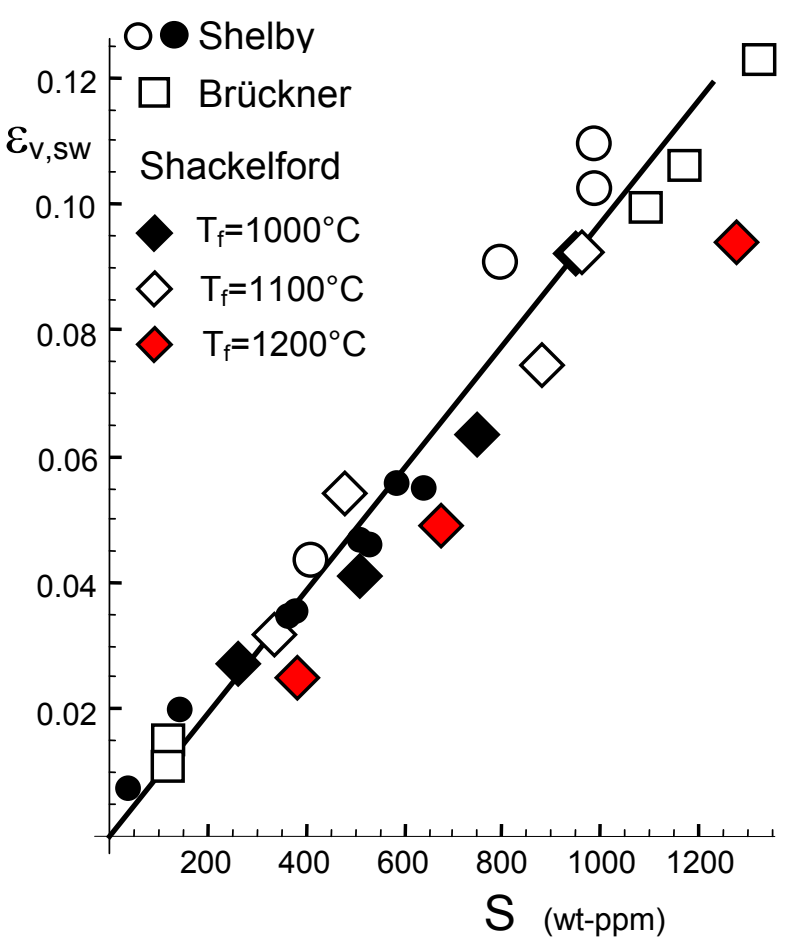

Fig. 1 Volume expansion strain vs. the hydroxyl concentration $S$, derived from density measurements compiled by Shelby [11]; line: eq.(8)).

Figure 1 represents the individual volume strains obtained from the measurements compiled by Shelby [11] as the symbols together with eq.(8) introduced as the straight line $[16,17]$.

On the basis of eq.(8), a positive sign of the activation volume in eq.(7) can be concluded from the Theorem of Le-Chatelier and Braun (principle of smallest constraint) $[3,10]$. For this purpose, let us consider a volume element in the glass (not necessarily located at the surface) that may contain a certain amount of water with concentration $C_{\mathrm{w}}$, e.g. introduced by diffusion. In order to ensure the condition of a "closed system", the volume may be surrounded by insulating walls which stop any escape or entrance of water and keep the total amount of water, $C_{\mathrm{w}}$, constant, Fig. $2 \mathrm{a}$. Depending on the temperature, the water $C_{\mathrm{w}}$ is divided into hydroxyl and molecular water according to eq.(4) with $k=k_{0}$. 
Experimental results for the equilibrium constant in the absence of stresses, $k_{0}$, were presented in reference [18] for the temperature range $90^{\circ} \mathrm{C} \leq \theta \leq 350^{\circ} \mathrm{C}$. These data were fitted by the empirical relation

$$
k_{0}=A \exp \left(-\frac{Q}{R T}\right)
$$

$\left(A=32.3\right.$ and $\left.Q=10.75 \mathrm{~kJ} / \mathrm{mol}, T=\left(\theta+273^{\circ} \mathrm{K}\right)\right)$.

a)



b)

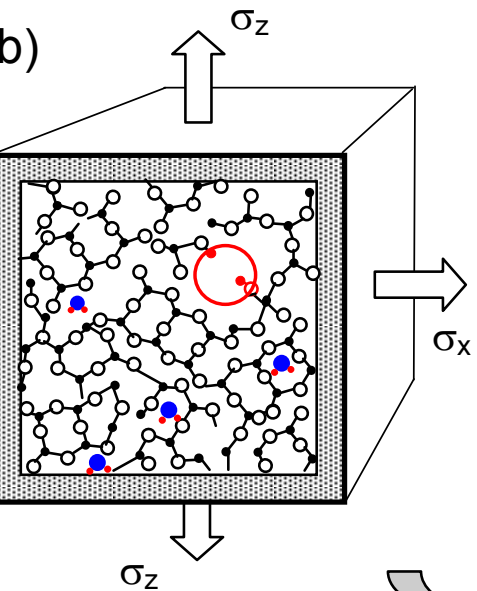

$\sigma_{\mathrm{z}}$
C) $\quad \varepsilon_{\mathrm{v}, \text { total }}=$ const.

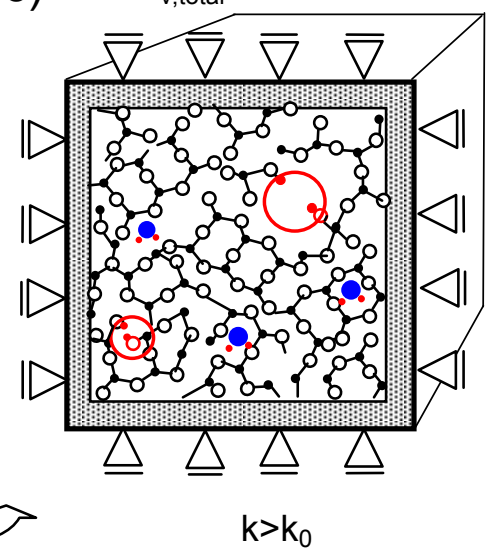

$\mathrm{k}>\mathrm{k}_{0}$

Equilibrium

Fig. 2 Volume element showing silica network, a) volume element with insulated bounderies suppresing water entrance or escape, b) stresses $\sigma_{x}=\sigma_{y}=\sigma_{z}$ applied to the volume element, c) state after reaching equilibrium of the water/silica reaction for total volume strain kept constant.

Applying tensile stresses $\sigma_{\mathrm{x}}=\sigma_{\mathrm{y}}=\sigma_{\mathrm{z}}$ abruptly on the volume element, Fig. 2b, the hydraulic stress component $\sigma_{\mathrm{h}}=\left(\sigma_{\mathrm{x}}+\sigma_{\mathrm{y}}+\sigma_{\mathrm{z}}\right) / 3=\sigma_{\mathrm{i}}$ causes an elastic volume increase, Fig. $2 \mathrm{~b}$, i.e. an elastic volume strain $\varepsilon_{\mathrm{v}, \text { elast }}$ that is given by

$$
\varepsilon_{v, \text { elast }}=\frac{3(1-2 v)}{E} \sigma_{h}
$$

where $E$ is Young's modulus and $v$ Poisson's ratio. Under constant displacement conditions, Fig. $2 \mathrm{c}$, this strain defines the total strain $\varepsilon_{\mathrm{v}, \text { total }}=$ const.

After reaching the equilibrium of the reaction (1), the total volume strain is the sum of elastic and swelling strains,

$$
\varepsilon_{v, \text { total }}=\varepsilon_{v, \text { last }}+\varepsilon_{v, s w}=\text { const }
$$

Since $\varepsilon_{\mathrm{V}, \mathrm{sw}}>0$, the initially applied elastic strain must decrease by $\varepsilon_{\mathrm{v}, \mathrm{sw}}$, i.e. the restrictions by the externally applied elastic strains decrease. Consequently, the stresses decrease, too. The Theorem of Le Chatelier [3] and Braun [10] then implies that the 
equilibrium must shift to the right side of eq.(1), i.e. that the reaction volume must be positive, $\Delta \bar{V}>0$. It is self-evident that this result must occur also for constant stress constraints, because any load-history can be described in the same way by dividing the load-application into several steps with constant total strains as in Fig. 2 with equilibrium of the reaction at the end of each substep.

It has to be emphasized that the Theorem of Le Chatelier and Braun allows qualitative statements only and cannot provide quantitative results. An estimate of the partial molar volume for $\mathrm{SiOH}, \Delta \bar{V}_{S}$, can be obtained directly from the data of Shelby [11], Shackleford [12] and Brückner [13, 14].

As outlined in $[16,17]: \Delta \bar{V}_{S}=7.52 \mathrm{~cm}^{3} /$ mole. Because two $\mathrm{SiOH}$ are created for each mole of water reacting, the total volume change per mole of water reacting is from the measurements at high temperatures: $\Delta \bar{V} \cong 2 \Delta \bar{V}_{S} \cong 15.03 \mathrm{~cm}^{3} /$ mole. When a significant amount of water is present, as is the case at lower temperatures, it hold

$$
\Delta \bar{V}=2 \Delta \bar{V}_{S}-\Delta \bar{V}_{C}
$$

with the reaction volume contribution of molecular water, $\Delta \bar{V}_{C}$. From $\Delta \bar{V}>0$ and $\Delta \bar{V}_{S}>0$, it yields $\Delta \bar{V}_{C}<2 \Delta \bar{V}_{S}$. This is one of the reasons why we neglected this volume in our former papers $[19,20]$.

\section{Negative reaction volume from literature}

All speculations on a negative reaction volume were caused by the interpretation of water concentration measurements on bending bars by Nogami and Tomozawa. In their conclusions, Nogami and Tomozawa [5] quoted: $\Delta \bar{V}<0$. A simple interpretation of this discrepancy was given in [8]. Here this point may be briefly addressed.

The experimental results by Oehler and Tomozawa [2], represented in Fig. 3, show a clear contradiction to the assumption of a constant surface value for the water concentration. There is, however, an incredible agreement with the thermal analogue of thermal shock behaviour under heat transfer boundary conditions, governed by a heat transfer coefficient. This calls for a surface condition

$$
\frac{d C}{d z}=\frac{h}{D}\left(C-C_{0}\right) \text { at } z=0
$$

where again $C_{0}$ is the concentration of molecular water reached at $z=0$ for $t \rightarrow \infty$.

Following the suggestion by Doremus [21], (Section 4.7), the phenomenological parameter $h$ in (14) may be interpreted as a reaction parameter for a slow surface reaction that limits the entrance of molecular water species.

On the other hand, a simpler phenomenological description is possible by assuming that a barrier exists to the transport of water across the surface of the glass. The barrier 
gives rise to a mass transfer coefficient for diffusion, which slows the passage of water into the glass. Each of the assumptions yields the same set of mathematical equations. The diffusivity for the case of stress-enhanced diffusion is given by the following equation [22]

$$
D=D_{0} \exp \left[\sigma_{h} \frac{\Delta V_{w}}{R T}\right]
$$

where $D_{0}$ denotes the value of the diffusivity in the absence of stresses. $T$ is the absolute temperature in $K ; \Delta V_{\mathrm{w}}$ is the activation volume for stress-enhanced diffusion and $R$ is the universal gas constant.

As shown by Carslaw and Jaeger [23] (Section 2.7), the concentration profile, $C(z)$ resulting from the boundary condition for a semi-infinite body is given by

$$
C(z, t) / C_{0}=\operatorname{erfc}\left[\frac{z}{2 \sqrt{D t}}\right]-\exp \left[\frac{h}{D} z+\frac{h^{2}}{D} t\right] \operatorname{erfc}\left[\frac{z}{2 \sqrt{D t}}+h \sqrt{\frac{t}{D}}\right]
$$

At the surface, $z=0$ :

$$
C(0, t) / C_{0}=1-\exp \left[\frac{h^{2}}{D} t\right] \operatorname{erfc}\left[h \sqrt{\frac{t}{D}}\right],
$$

In Fig. 3, the molecular water concentration at the surface is plotted versus the time with the concentration normalized on the saturation value.

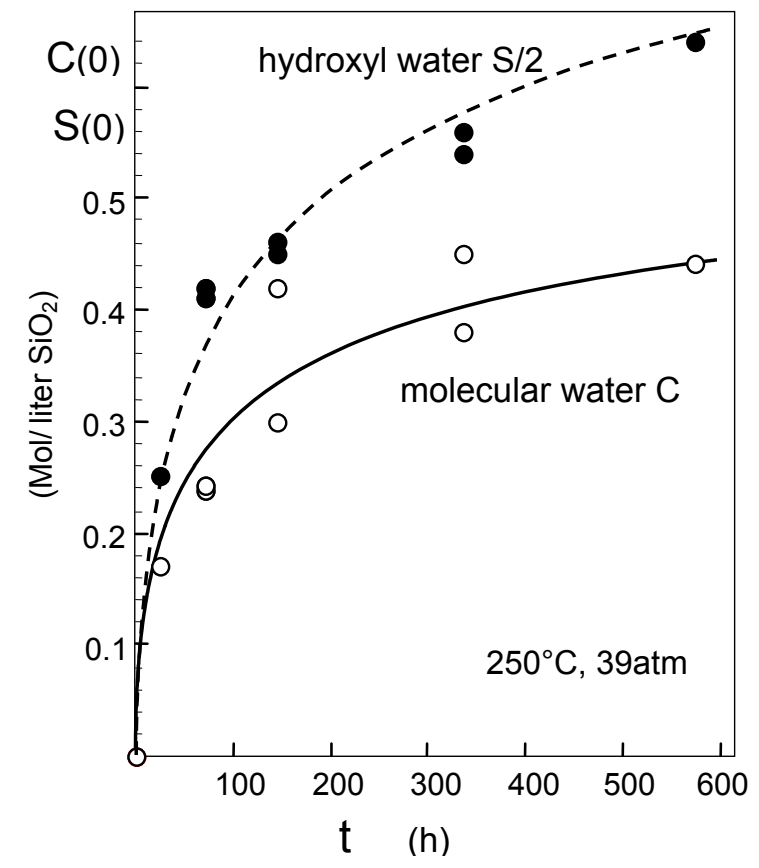

Fig. 3 Molecular water $(C)$ and hydroxyl water content $(S / 2)$ at the surface of silica for $250^{\circ} \mathrm{C}$ at an equilibrium vapour pressure of $39 \mathrm{~atm}$ from Oehler and Tomozawa [2]. 

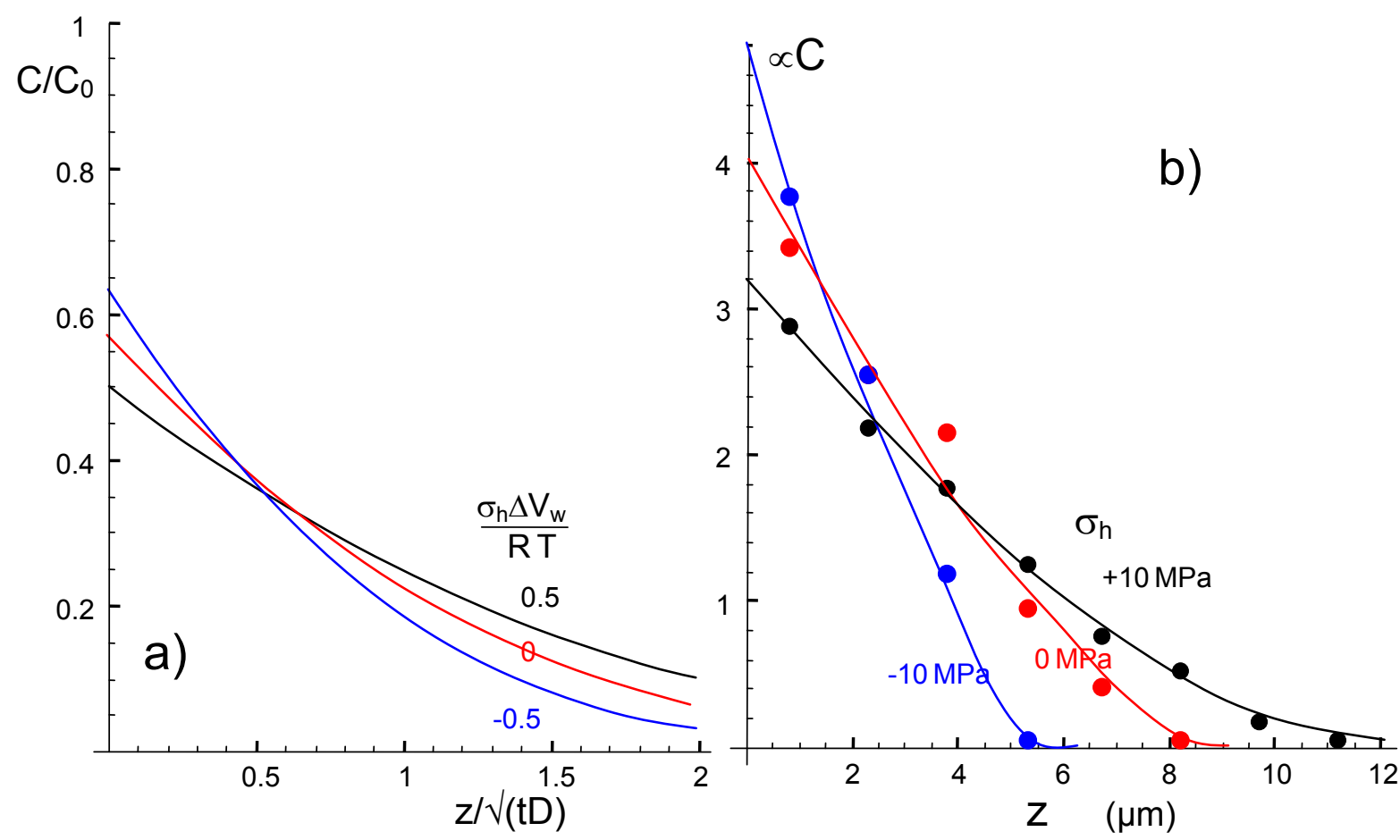

Fig. 4 a) Diffusion profiles from eq.(16) under externally applied stresses for a soaking time of $t h^{2} / D=1, \mathrm{~b}$ ) Concentration profiles by Nogami and Tomozawa [5] as a function of the hydrostatic stress for bending bars at $192^{\circ} \mathrm{C}$ (water vapour pressure: 12.3 bars).

Concentration profiles computed with eq.(16) are plotted in Fig. 4a as a function normalized depth $z$ for a normalized time $t h^{2} / D=1$. It is obvious that the surface concentration is smaller under externally applied tensile stresses than in the absence of such stresses. Compressive stresses cause an increase of hydroxyl water at the surface. The higher surface concentration for compression decreases with a steeper profile than under tension. In deeper regions the concentrations under tension exceed those for the stress-free and the compression case.

The computed water profiles and the measurements by Nogami and Tomozawa [5], Fig. $4 \mathrm{~b}$, agree excellently. This makes clear that the apparently increased water solubility under compression is an artifact of the stress-dependent diffusivity, eq.(15).

In words: Due to the higher diffusivity in tension, the water at the tensile surface can drain easier into the bulk than in the compression state. Because of the restricted water entrance for a finite mass transfer coefficient, $h<\infty$, the concentration must decrease at the tensile surface and increase on the compression surface. This is a consequence of the stress-dependent diffusivity and must not be understood as an enlarged solubility of water under compressive stresses.

\section{Summary}

We applied the Theorem by Le Chatelier [3] and Braun [10] to the problem of the stress-dependent equilibrium of the water/silica reaction in an arbitrary volume ele- 
ment that contains a fixed amount of water. It was found that the externally applied constraints, here the elastic tensile strains, are reduced by the positive swelling strains that are proportional to the hydroxyl concentration $S$. Consequently, the reaction must be forced into the direction of an increased hydroxyl content; with other words, the reaction volume must be positive.

\section{References}

1 Doremus RH. Diffusion of water in silica glass. J Mater Res. 10;1995:2379-2389.

2 Oehler A, Tomozawa M. Water diffusion into silica glass at a low temperature underhigh water vapor pressure. J Non-Cryst Solids. 347;2004:211-219.

3 Le Chatelier H, CR Acad Sci Paris. 99;1884:786.

4 Hamann SD. High Pressure Physics and Chemistry, Ed. Bradley RS. Academic Press, London. 1963:163-207.

5 M. Nogami, M. Tomozawa, Diffusion of water in high silica glasses at low temperature, Phys. and Chem. of Glasses 25(1984), 82-85.

6 A. Agarwal, M. Tomozawa, W.A. Lanford, Effect of stress on water diffusion in silica glass at various temperatures, J Non-Cryst Solids. 167;1994:139-148.

7 S.W. Freiman, S.M. Wiederhorn, J.J. Mecholsky, Environmentally Enhanced Fracture of Glass: A Historical Perspective, J Am Ceram Soc. 92, 2009, 1371-82.

8 S. M. Wiederhorn, G. Rizzi, G. Schell, S. Wagner, M. J. Hoffmann, T. Fett, Diffusion of water in silica: Influence of moderate stresses, J. Am. Ceram. Soc. 101(2015), 1180-1190.

9 M. Tomozawa, K. M. Davis, J. H. Seaman, E. M. Aaldenberg The origin of anomalous water diffusion in silica glasses at low temperatures, J Am Ceram Soc. 100, 2017, 4548-4561.

10 F. Braun, Untersuchungen über die Löslichkeit fester Körper und die den Vorgang der Lösung begleitenden Volum- und Energieänderungen, Ztschr. f. phys. Chem. 1. (1887), 259272.

11 Shelby JE. Density of vitreous silica. J Non-Cryst Solids. 349;2004:331-336.

12 Shackelford JF, Masaryk JS, Fulrath RM. Water Content, Fictive Temperature and Density Relations for Fused Silica. J Am Ceram Soc. 53;1970:417.

13 Brückner R. The structure-modifying influence of the hydroxyl content of vitreous silicas. Glastech Ber. 43;1970:8-12.

14 Brückner R. Metastable equilibrium density of hydroxyl-free synthetic vitreous silica. $J$ Non-Cryst Solids. 5;1971:281-285.

15 S. M. Wiederhorn, M. J. Hoffmann, T. Fett, Swelling strains from density measurements, Scientific Working Papers 38, 2015, ISSN: 2194-1629, Karlsruhe, KIT.

16 S. M. Wiederhorn, T. Fett, K.G. Schell, Molar volume of SiOH estimated from swelling strains, to be submitted to J Am Ceram Soc. 
17 T. Fett, K.G. Schell, S.M. Wiederhorn, Swelling strains from gamma-irradiated silica Evaluation of results by Shelby, Scientific Working Papers 103, 2015, ISSN: 2194-1629, Karlsruhe, KIT.

18 Wiederhorn SM, Yi F, LaVan D, Fett T, Hoffmann MJ. Volume expansion caused by water penetration into silica glass. J Am Ceram Soc. 98;2015:78-87.

19 S.M. Wiederhorn, T. Fett, G. Rizzi, M. Hoffmann, J.-P. Guin, "Water Penetration - its Effect on the Strength and Toughness of Silica Glass," Met. Mater. Trans. A, 44 (2013) [3], $1164-1174$.

20 T. Fett, G. Rizzi, M. Hoffmann, S. Wagner, and S.M. Wiederhorn, "Effect of Water on the inert Strength of Silica Glass: Role of Water Penetration," J. Am. Ceram. Soc. 95 (2012) [12], 3847-3853.

21 R.H. Doremus, Diffusion of Reactive Molecules in Solids and Melts, Wiley, 2002, NewYork.

22 P.G. Shewman, Diffusion in Solids, McGraw-Hill, New York, 1963.

23 Carslaw, H.S., Jaeger, J.C. Conduction of heat in solids, 2nd ed. 1959, Oxford Press, London. 
KIT Scientific Working Papers

ISSN 2194-1629

www'kit.edu 